Revue Gouvernance

Governance Review

GOUVERNANCE

GOU ERNAN CLEm

\title{
Démocratie constructive et gouvernance de la technique
} Les conditions de la gouvernance démocratique dans un processus technique et social complexe : l'exemple du projet européen Cowam-in-Practice dans la gestion des déchets radioactifs

\section{Constructive democracy and the governance of technology}

\author{
Sylvain Lavelle, Gilles Hériard Dubreuil, Serge Gadbois, Claire Mays et Thierry \\ Schneider
}

Volume 7, numéro 2, 2010

URI : https://id.erudit.org/iderudit/1038920ar

DOI : https://doi.org/10.7202/1038920ar

Aller au sommaire du numéro

Éditeur(s)

Centre d'études en gouvernance de l'Université d'Ottawa

ISSN

1912-0362 (numérique)

Découvrir la revue

Citer cet article

Lavelle, S., Hériard Dubreuil, G., Gadbois, S., Mays, C. \& Schneider, T. (2010). Démocratie constructive et gouvernance de la technique : les conditions de la gouvernance démocratique dans un processus technique et social complexe : l'exemple du projet européen Cowam-in-Practice dans la gestion des déchets radioactifs. Revue Gouvernance / Governance Review, 7(2).

https://doi.org/10.7202/1038920ar
Résumé de l'article

Il existe au sein des théories et des pratiques de la gouvernance démocratique une tendance dominante qui trouve son origine dans la pensée dialogique contemporaine, celle de Habermas en particulier, et dans une moindre mesure, celle de Latour et Callon. Les modèles dialogiques se distinguent par le privilège qu'ils accordent à des degrés divers à la discussion et à l'entente, au risque de favoriser les dispositifs institutionnels de démocratie délibérative et participative, tels que le débat public ou la conférence de citoyen. Le modèle de la démocratie constructive apparaît en comparaison comme une voie alternative qui permet de combler certaines lacunes des modèles dialogiques. Le modèle de la démocratie constructive peut s'appliquer au sein de la gouvernance de la technique dans les secteurs où la tendance dialogique est manifeste et où elle tend à conforter une forme de « technocratie éclairée ». Le dispositif du projet européen Cowam-in-Practice (CIP) propose une expérimentation inspirée par la démocratie constructive dans la gestion des déchets radioactifs, issus pour l'essentiel de l'exploitation des centrales nucléaires. La voie de la démocratie constructive mise à l'épreuve dans ce processus technique et social complexe vise une transformation du système de relations entre acteurs ainsi qu'une consolidation de l'autonomie tant heuristique que stratégique de la société civile. 


\title{
Démocratie constructive et gouvernance de la technique : Les conditions de la gouvernance démocratique dans un processus technique et social complexe : l'exemple du projet européen Cowam-in-Practice dans la gestion des déchets radioactifs
}

\author{
Par Sylvain Lavelle, Gilles Hériard Dubreuil, Serge Gadbois, Claire Mays et Thierry Schneider
}

Résumé : Il existe au sein des théories et des pratiques de la gouvernance démocratique une tendance dominante qui trouve son origine dans la pensée dialogique contemporaine, celle de Habermas en particulier, et dans une moindre mesure, celle de Latour et Callon. Les modèles dialogiques se distinguent par le privilège qu'ils accordent à des degrés divers à la discussion et à l'entente, au risque de favoriser les dispositifs institutionnels de démocratie délibérative et participative, tels que le débat public ou la conférence de citoyen. Le modèle de la démocratie constructive apparaît en comparaison comme une voie alternative qui permet de combler certaines lacunes des modèles dialogiques. Le modèle de la démocratie constructive peut s'appliquer au sein de la gouvernance de la technique dans les secteurs où la tendance dialogique est manifeste et où elle tend à conforter une forme de «technocratie éclairée ». Le dispositif du projet européen Cowam-in-Practice (CIP) propose une expérimentation inspirée par la démocratie constructive dans la gestion des déchets radioactifs, issus pour l'essentiel de l'exploitation des centrales nucléaires. La voie de la démocratie constructive mise à l'épreuve dans ce processus technique et social complexe vise une transformation du système de relations entre acteurs ainsi qu'une consolidation de l'autonomie tant heuristique que stratégique de la société civile.

\section{Introduction}

Il existe des domaines de la technique qui ne sont pas spontanément enclins à une forme d'ouverture démocratique à la société, pour des raisons liées à leur développement historique, ou à la nature et la complexité de leur activité. L'expertise et la décision s'y concentrent d'ordinaire sur les aspects techniques, bien que ces domaines d'activité comportent et articulent des aspects doublement techniques et sociaux. C'est ainsi que l'inclusion en leur sein de la « société civile », sous la forme d'une coopération avec des citoye ns ou des associations, ne va pas toujours de soi. C'est le cas, par exemple, dans les domaines d'activité sensibles que sont les secteurs de l'industrie nucléaire ou de l'industrie pétrolière, pour ne citer qu'eux. Il existe toutefois une multitude d'initiatives contemporaines en matière de gouvernance des activités techniques qui témoignent d'un changement progressif d'état d'esprit. Elles vont dans le sens d'une prise en compte accrue d'aspects de ces activités qui pendant longtemps ont été confiés aux seuls experts et décideurs.

Dans le champ assez vaste de la gouvernance démocratique, certains modèles issus de la philosophie ou de la sociologie, notamment ceux de Habermas et de Latour et Callon, occupent une place dominante. Ces modèles relevant de la démocratie délibérative ou participative fonctionnent comme un cadre de référence censé être valable tant pour les conceptions théoriques que pour les expérimentations pratiques de la gouvernance. Or, il est notable qu'il s'agit pour l'essentiel de modèles dialogiques qui privilégient, à des degrés divers selon les auteurs et courants, la voie de la discussion démocratique et la visée d'entente cognitive et 
normative. Le dialogisme se manifeste soit sous la forme d'un procéduralisme fort à la Habermas, soit sous la forme d'un procéduralisme faible à la Latour et Callon. Ainsi, le tropisme procédural de ces modèles favorise un type de dispositif dialogique plus ou moins institutionnel, dans le genre du débat public ou de la conférence de citoyens. Il est cependant manifeste que la procédure dialogique échoue en de nombreuses circonstances sur l'écueil des antagonismes ou des asymétries multiples qui affectent ces dispositifs ponctuels de discussion démocratique ${ }^{1}$. Il convient donc de prendre acte des limites manifestes de ces modèles dialogiques jusqu'ici dominants, ainsi que des dispositifs dialogiques qui en découlent, sans pour autant jeter le bébé avec l'eau du bain.

Ainsi, il ne s'agit pas de se livrer à une condamnation sans appel de ces modèles et dispositifs dialogiques, mais d'opter pour une méthode permettant une discrimination (au sens de « jugement critique ») du potentiel d'inclusion que recèle tel ou tel type de processus démocratique. Il peut être intéressant et utile à cet effet de présenter un ensemble de conceptions et d'expérimentations développées en Europe dans le domaine sensible, touchant aussi bien la science, la société que l'environnement, de la gouvernance de l'industrie nucléaire. L'un de ces dispositifs en particulier, le projet européen Cowam-In-Practice (CIP), s'inscrit dans une réflexion européenne sur la gouvernance des activités de gestion des déchets radioactifs issus pour l'essentiel de l'exploitation des centrales nucléaires. Il retient l'attention par sa détermination à proposer une voie alternative en matière de gouvernance démocratique et de recherche coopérative.

Ce genre de dispositif mêlant une variété d'acteurs (experts, décideurs publics et privés, citoyens, associations, etc.) propose un modèle de démocratie qui ne relève pas de la démocratie représentative, ni d'ailleurs de la démocratie délibérative ou participative à proprement parler. Il s'agit en fait d'un modèle de démocratie constructive qui propose de mettre en oævre, au moyen d'un dispositif institutionnel et expérimental transitoire, les conditions d'une gouvernance visant une réflexion et une coopération durable entre acteurs ${ }^{2}$.

\section{Typologie des modèles de démocratie}

Le modèle de la démocratie constructive ne constitue pas en soi une alternative radicale à l'égard des modèles de la démocratie délibérative et de la démocratie participative. Il fonctionne plutôt comme un modèle complémentaire qui propose de combler certaines lacunes des modèles dialogiques, en particulier certains des «points aveugles» des dispositifs institutionnels. La démocratie délibérative repose sur le principe de la discussion, laquelle peut éventuellement être articulée jusqu'à un certain point à la décision. La démocratie participative repose quant à elle sur le principe de l'inclusion, laquelle peut être selon les conceptions une inclusion à la discussion ou à la décision, éventuellement dans un cadre ponctuel ou permanent, au sein d'une

\footnotetext{
${ }^{1}$ De ce point de vue, le débat public mené récemment en France sur le thème de l'avenir des nanosciences et des nanotechniques est un exemple éclatant de l'échec de la procédure dialogique de débat public dans sa version institutionnelle, de type CNDP (Commission Nationale du Débat Public). Il s'agit manifestement d'un genre de dispositif qui n'est pas adapté àce genre de débat.

2 Cette conception relève plus d'un constructivisme démocratique que d'un dialogisme démocratique (Habermas, Latour, Callon) ou même d'un expérimentalisme démocratique (Sabel, Cohen) - même si le dialogue et l'expérimentation apparaissent par la suite comme des ingrédients d'un processus de gouvernance démocratique.
} 
organisation ou d'une institution. Ces deux formes de démocratie ont toutefois le défaut de proposer des dispositifs dialogiques sans s'appesantir sur leurs conditions d'arrière-plan, qui peuvent être en l'espèce des conditions non-dialogiques. C'est précisément cette lacune que propose de combler l'approche en termes de démocratie constructive, laquelle se réclame d'un modèle de gouvernance réflexive et coopérative durable.

\section{Les modèles de la démocratie délibérative et participative}

On peut tout d'abord rappeler les principaux aspects des modèles de la démocratie délibérative et participative, du moins tels qu'ils ont été définis et précisés part David Held dans son ouvrage désormais classique, Models of democracy ${ }^{3}$. De façon générale, la démocratie délibérative et participative relèvent du modèle dialogique, en ceci, qu'elles se distinguent toutes deux d'une conception agrégative de la formation de la volonté générale.

La démocratie délibérative, selon Held, a pour principe de justification le fait que les termes et conditions de l'association politique procèdent de l'assentiment libre et raisonné des citoyens. En outre, la possibilité d'une justification mutuelle des décisions politiques est la base légitime pour trouver des solutions aux problèmes collectifs. Elle possède plusieurs caractéristiques saillantes: sondages délibératifs, journées délibératives, jurys citoyens, initiatives de gouvernement électronique (du rapport en ligne à l'accès direct aux représentants), programmes de démocratie électronique incluant des forums publics en ligne, analyse de groupe et production de propositions politiques, délibération à travers la vie publique, du micro-forum au dispositif transnational, nouvel usage des referendums liés aux sondages délibératifs, etc. La démocratie délibérative, selon Held, a pour conditions générales le pluralisme des valeurs, un programme fort d'éducation civique, une culture et des institutions publiques soutenant le développement de préférences «reconsidérées » et « réfléchies », un financement public de corps et de pratiques délibératives, et d'associations secondaires qui les soutiennent.

La démocratie participative, en comparaison, est fondée sur le principe de justification suivant : un droit égal à la liberté et au développement de soi peut être réalisé seulement dans une société participative. C'est une société qui favorise le sens de l'efficacité politique, nourrit l'attention pour les problèmes collectifs, et contribue à la formation d'une citoyenneté réfléchie capable de manifester un intérêt soutenu pour le processus du gouvernement. Elle possède des caractéristiques saillantes, telles que la participation directe des citoyens dans la régulation des institutions-clés de la société, incluant le lieu de travail et la communauté locale, la réorganisation du système de parti qui rend les dirigeants de parti directement responsables (accountable) auprès des membres, l'organisation d'événements participatifs (participatory parties) au sein de la structure parlementaire, l'entretien d'un système institutionnel ouvert garantissant la possibilité d'expérimentation de formes politiques. Elle a pour conditions générales l'amélioration directe de la base de ressources de beaucoup de groupes sociaux à travers la redistribution de ressources matérielles, la réduction (et si possible, éradication) du pouvoir bureaucratique dans la vie publique et privée, un système d'information ouvert afin de garantir des décisions informées, un réexamen des allocations de soin pour les enfants afin que les femmes et les hommes puissent participer à la vie publique.

\footnotetext{
${ }^{3}$ David Held (1993).
} 
Il est indéniable que les deux modèles recèlent des différences substantielles ${ }^{4}$, et même parfois des tensions ${ }^{5}$, mais ils se recoupent au moins en ceci, qu'ils relèvent tous deux, à un degré plus ou moins poussé, d'un modèle dialogique de la démocratie.

\section{Les dimensions harmonique et agonistique des modèles}

Les modèles de la démocratie dialogique sont multiples et se situent a priori plutôt du côté de la démocratie délibérative, encore qu'ils empruntent parfois quelques éléments à la démocratie participative. C'est le cas notamment lorsqu'il est question, d'une façon que certains pourront juger acuménique, de modèles ou dispositifs dialogiques de «participation délibérative ». On peut donner une idée de la variété de ces modèles dialogiques en pointant la référence plus ou moins marquée à l'égard de la notion de situation idéale de parole. Ils se répartissent ainsi entre une tendance «harmonique » fondée sur l'entente et une tendance plus «agonistique » qui reconnaît le conflit. De ce point de vue, le modèle dialogique de Habermas est clairement orienté vers l'entente, tandis que ceux de Latour et Callon paraissent, en comparaison, réserver une place plus grande au conflit ${ }^{6}$.

Ainsi, le modèle de la discussion «à la Habermas » suggère une différence entre, d'une part, l'activité dialogique orientée vers l'entente et, d'autre part, l'activité technique et stratégique orientée vers le succès ou la performance ${ }^{7}$. Les idéalisations contrefactuelles fortes de la procédure de discussion sont maintenues par Habermas jusqu'au bout, envers et contre toutes les critiques. Ainsi, ce dernier identifie quatre idéalisations fondamentales qui sont présupposées selon lui dans l'agir communicationnel ${ }^{8}$, de même que quatre présuppositions pragmatiques, dont celles d'entente, d'inclusion, d'égalité, de sincérité et d'absence de contraintes ${ }^{9}$. Or, la démocratie ne consiste pas seulement en la recherche de consensus, mais peut emprunter à l'occasion la voie du conflit, lequel permet aussi de faire émerger à certaines conditions un

\footnotetext{
${ }^{4}$ Blondiaux (2008, p. 44) suggère que «théories de la démocratie participative et de la démocratie délibérative partagent de nombreux présupposés communs, en particulier l'insatisfaction à l'égard d'une définition de démocratie et de la citoyenneté limitée au principe majoritaire et au seul accomplissement du vote. Elles se croisent sans cesse. Elles se distinguent cependant par leur finalité principale. Les premières recherchent la formation de communautés citoyennes actives, mettent l'accent sur l'engagement et la politisation des participants. Les secondes visent à mieux fonder la décision politique en liant cette dernière à un processus d'argumentation rationnelle impliquant des points de vue contradictoires. Si la distinction entre ces perspectives, entre ces finalités possibles de la participation, a été parfois exagérée, elle n'en est pas moins réelle ».

5 On peut se reporter notamment pour l'examen des tensions entre démocratie délibérative et démocratie participative à l'article de Cohen et Fung (2004).

6 L'alternative la plus nette au modèle de Habermas (1991 et 1996), qui demeure toutefois dans le cadre dialogique, se trouve dans la conception inclusive de la démocratie délibérative préconisée par Young (2000). Celle-ci ne se limite pas, en effet, à l'usage d'arguments, mais inclut des modalités de persuasion et de narration et n'exclut pas le conflit ni le désordre dans la discussion.

7 Jürgen Habermas (1987).

8 Jürgen Habermas (2006) p. 20-21.

9 idem, p. 56, (1) la publicité et l'inclusion : quiconque peut apporter une contribution pertinente à la controverse dont une prétention à la validité est l'objet ne peut être exclu. (2) l'égalité des droits dans la communication : tous reçoivent les mêmes chances de s'exprimer sur la question débattue. (3) l'exclusion de toute mystification ou illusion : les participants doivent penser ce qu'ils disent. (4) l'absence de contrainte : la communication doit être affranchie de toute restriction susceptible l'empêcher la manifestation du meilleur argument et de déterminer l'issue de la discussion.
} 
potentiel de coopération entre acteurs ${ }^{10}$. En outre, la pratique de la démocratie articule en permanence l'agir dialogique et l'agir stratégique, tandis que dans le modèle théorique de la discussion de Habermas, la stratégie assimilée à une forme de manipulation n'a tout simplement pas sa place $^{11}$.

Le modèle de la discussion de Latour et Callon, voisin de celui de Habermas, relève de la démocratie dialogique en général et de la démocratie technique en particulier ${ }^{12}$. Il est arrimé à une procédure de dialogue qui reconnaît la place du conflit et de la manoæivre, ce qui le différencie nettement du modèle de Habermas ${ }^{13}$. Nous supposons qu'il existe un modèle commun de la discussion chez Latour et Callon, bien qu'il ne soit pas exprimé dans une forme aussi explicite que celui de Habermas. La caractéristique de ce modèle de la discussion est qu'il ajoute à l'argumentation l'enjeu de la traduction, et à la visée d'entente celle de reconnaissance et de co-production. Cependant, il peine à concevoir l'organisation de la montée en compétence, en puissance et en réflexivité durable de la société civile, autrement que comme un développement nécessaire du réseau et de la traduction. En un sens, aux écueils du procéduralisme fort de Habermas répondent les écueils du procéduralisme faible de Latour et Callon.

On peut tenter sous une forme schématique une comparaison des deux modèles en faisant ressortir leurs points de convergence ainsi que leurs points de divergence :

\section{Modèle dialogique (D1) : Habermas}

(1) Les partenaires d'une communauté de communication $C$

(2) Jouissant des conditions de symétrie de l'échange $S$

(3) Utilisent les moyens de l'argumentation $A$

(4) Visent la fin de l'entente $E$

Modèle dialogique (D2) : Latour/Callon

(1) Les partenaires d'une communauté de communication $C$

(2) Jouissant ou non des conditions de symétrie de l'échange $S$

(3) Utilisent les moyens de l'argumentation $A$, ainsi que de la traduction $T$ et de l'apprentissage

A

(4) Visent la fin de l'entente $E$, de la reconnaissance $R$, ou de la production commune $P$ (coproduction)

\footnotetext{
${ }^{10}$ Georg Simmel (1995), ainsi que Iris Young (2000).

${ }_{11}$ Pour une critique de ce modèle, voir Axel Honneth (2000), ainsi que Sylvain Lavelle (2007).

12 On pourrait rapprocher afin d'en montrer les convergences le modèle de démocratie technique de Callon, Lascoumes et Barthe (2001) des modèles proposés par Winner (2002), Sclove (2003) et Feenberg (2004).

13 Il est fait explicitement mention de procédures de dialogue chez Latour (1999), Callon (1998) et Callon, Lascoumes et Barthe (2001). La démocratie dialogique apparaît comme une solution aux lacunes de la démocratie délégative (la double délégation- aux experts et aux élus), en particulier parce qu'elle rend possible la recherche d'un monde commun au moyen de forums hybrides. «Les procédures dialogiques (...) sont destinées à organiser une recherche coopérative entre spécialistes et profanes » (Callon et al., 2001, p.340).
} 
S'il est jugé illégitime d'associer de façon «positive» Callon et Latour dans un modèle dialogique unique, tout du moins, il paraît légitime de les associer de façon «négative », ie en rappelant ce qui le distingue du modèle dialogique de Habermas ${ }^{14}$.

\section{La critique des modèles dialogiques de la démocratie}

Il est notable que les modèles dialogiques ont été une source d'inspiration pour une multitude de dispositifs dialogiques de délibération et de participation. Or, ces dispositifs reflètent un certain nombre de faiblesses qui sont inhérentes à ces modèles dialogiques.

Historiquement, la délibération ou la participation institutionnelle intervient en général assez tard dans le processus de construction de la décision, parfois même après la décision, ou lorsque les principales options sont déjà fixées ${ }^{15}$. Elle vise alors surtout à favoriser l'acceptabilité sociale de la décision et à rassurer les acteurs de la société quant à la qualité des décisions prises par les décideurs. Certains mécanismes institutionnels ont tenté de favoriser une délibération ou une participation plus en amont de la décision, notamment pour répondre à des critiques concernant l'absence de lien avec la décision ${ }^{16}$. Cependant, d'une façon générale, ces processus sont impuissants à résoudre le déficit démocratique qui est inhérent aux mécanismes de gouvernance traditionnels. Cette gouvernance traditionnelle est fondée, d'une part, sur des dispositifs de représentation démocratique et, d'autre part, sur des formes de rationalisation des décisions élaborées par des structures technocratiques (des experts et des serviteurs de l'Etat). Il s'agit alors d'intégrer le bien commun dans une forme rationalisée d'intérêt général et de construire des décisions pour la population, et non pas avec celle-ci.

Au sein des dispositifs dialogiques, la délibération ou la participation ne vise pas à transformer les processus de décision existants, mais plutôt à les conforter, ou à surmonter des situations de blocage, sans changer les formes de gouvernance établies. Il est notable que ces processus de délibération ou de participation sont rarement fondés sur l'idée d'une inclusion permanente, ou d'une inclusion durable de la société. Ils se fondent plutôt sur l'ouverture de fenêtres ponctuelles de délibération et de participation, et sur une visée de clôture du processus une fois atteint un certain niveau d'acceptabilité sociale. Or, ces dispositifs sont mis à l'épreuve par la tendance manifeste à la désaffection des acteurs de la société, lorsqu'ils ne sont pas porteurs de potentialités réelles de changement de la gouvernance. La désaffection sociale est imputée à la fameuse stakeholder fatigue (ou « fatigue des porteurs d'enjeux »), laquelle ne fait que traduire en réalité un certain scepticisme, voire une frustration, et même parfois un rejet vis-à-vis de processus qui n'ont que l'apparence du changement.

\footnotetext{
${ }^{14}$ Pour une présentation conjointe des modèles de Habermas et de Latour et Callon, on peut se reporter à Lavelle (2006).

${ }^{15}$ C'est le cas en France, par exemple, lors des débats publics institutionnels de type CNDP (Commission Nationale du Débat Public) portant sur le projet de construction d'un réacteur nucléaire dit de nouvelle génération. La décision de construire le réacteur EPR de Flamanville avait déjà été prise par le pouvoir politique (le gouvernement Raffarin) avant le lancement du débat public, ce qui a fortement limité l'intérêt des discussions dans ce débat public, qui du reste a été confronté à de fortes difficultés de mise en oeuvre.

16 Voir Revel et al. (2007).
} 
Les limites des conceptions dialogiques de la démocratie ont bien été identifiées par les partisans d'une gouvernance réflexive, en particulier Lenoble et Maesschalck ${ }^{17}$. Ils qualifient la procédure dialogique de Latour et de Callon de procéduralisme faible et celle de Habermas, en comparaison, de procéduralisme fort ${ }^{18}$. L'idée de la gouvernance réflexive est de favoriser la déstabilisation des cadres cognitifs et normatifs des acteurs qui conditionnent leur engagement dialogique ainsi que leur cadrage problématique. L'enjeu de cette déstabilisation (la modalisation) est qu'ils puissent poser différemment les problèmes identifiés dans le processus de gouvernance, tant sur le plan épistémique ou technique que sur le plan éthique ou politique. C'est aussi de favoriser une montée en capacité (la capacitation) ainsi qu'une réflexivité de second ordre, définie par la prise de distance à l'égard de la procédure et du processus de gouvernance lui-même; elle se distingue d'une réflexivité de premier ordre, centrée sur les conséquences des activités.

Les modèles de gouvernance démocratique, en particulier les modèles dialogiques, présentent selon Lenoble et Maesschalck deux limites fondamentales :

(1) Les conditions de réalisation de l'exigence démocratique sont supposées 'formalisables' et intégrables dans un aménagement institutionnel de représentation de la volonté souveraine des membres du groupe.

(2) La réflexivité qui s'exprime au travers du processus d'interaction présupposerait données les deux parties de ce travail d'interaction, c'est-à-dire la société et le pouvoir.

Sur ce deuxième point, Lenoble et Maesschalck soulignent l'absence de construction de la relation entre la société et le pouvoir dans les conceptions contemporaines de la démocratie, notamment la démocratie d'interaction de Rosanvallon ${ }^{19}$ :

\begin{abstract}
«Tout semble...se passer...comme si le 'social' était d'emblée toujours déjà donné. La réflexivité qui s'exprimerait ainsi au travers du processus d'interaction présupposerait données les deux parties de ce travail d'interaction, c'est-à-dire la société et le pouvoir...Tout semble donc se passer comme si la seule convocation des acteurs, voire leur multiplication ou leur simple agrégation, suffisait à assurer la production d'une représentation aussi adéquate que possible des intérêts sociaux en présence et à permettre ainsi, par le simple jeu d'une confrontation aux propositions du pouvoir, d'assurer, par une forme de capacité réflexive spontanée, la construction, en devenir constant, de la représentation collective du vouloir commun ».
\end{abstract}

La gouvernance réflexive suppose qu'un dispositif de gouvernance démocratique doit pouvoir construire les conditions d'une émergence des acteurs sociaux ainsi que les conditions de la réflexivité qu'ils peuvent développer dans les processus. Elle donne une idée, ne serait-ce que par l'identification des lacunes propres aux conceptions de la délibération et de la participation, de ce que peut être en comparaison une démocratie constructive.

\title{
Le modèle de la démocratie constructive
}

\footnotetext{
${ }^{17}$ Lenoble et Maesschalck (2010 et 2009).

${ }^{18}$ Sur la différence entre les deux versions du procéduralisme, voir Lenoble et Maesschalck (2009).

${ }^{19}$ Lenoble et Maesschalck (2009) p. 30-31.
} 
Par démocratie constructive, on peut entendre une forme de démocratie dans laquelle la gouvernance coopérative, réflexive et durable entre acteurs nécessite une véritable construction des activités et des capacités des citoyens, tant d'un point de vue logistique, heuristique que stratégique. La notion de démocratie constructive rend également compte de la construction d'une culture démocratique de la coopération qui a l'ambition d'être durable, dans laquelle les acteurs peuvent aborder les questions techniques parfois pointues avec leur regard légitime de citoyen $^{20}$.

La démocratie constructive, si elle recourt à des dispositifs dialogiques, ne se confond pas avec la démocratie dialogique, car elle articule les conditions dialogiques avec les conditions nondialogiques de la gouvernance. Parmi ces conditions non dialogiques, on trouve, aut-delà des conditions discursives, des conditions d'arrière-plan d'ordre culturel, mental, corporel, organisationnel, institutionnel, matériel, financier, etc. ${ }^{21}$. De plus, la démocratie constructive se démarque des modèles désormais classiques de la démocratie délibérative et participative, en particulier de leur modalité institutionnelle. En effet, la construction de conditions d'arrière-plan permet de faire émerger de nouvelles catégories d'acteurs de la société civile, en posture de compétence et de puissance au sein des dispositifs délibératifs ou participatifs. Cependant, la démocratie cons tructive n'est pas exclusive d'autres formes aujourd'hui canoniques de démocratie (représentative, participative, délibérative) et en cela peut parfaitement s'accommoder d'une constitution mixte.

Parmi les conditions d'arrière-plan de la démocratie constructive, on peut citer le rôle majeur et spécifique que peuvent jouer les «agences démocratiques de support et d'expertise », qui vont plus loin dans leur rôle que les agences de veille citoyenne ${ }^{22}$. Le but de ces agences démocratiques est de mettre en ouvre les moyens logistiques, heuristiques et stratégiques qui vont permettre à terme de faire accéder la société civile à une autonomie. L'idée qui se trouve derrière ces agences est que l'autonomie acquise au moyen d'une montée en compétence et en puissance des acteurs sociaux doit être construite par une organisation démocratique qui se met pour ainsi dire au service de la société civile.

${ }^{20}$ Il s'agit en fait d'un véritable partenariat coopératif qui peut impliquer une co-expertise, de même éventuellement qu'une contre-expertise diligentée par la société civile si besoin est. De ce point de vue, l'appropriation d'une culture technique est un ingrédient essentiel du développement de la démocratie constructive.

${ }^{21}$ Le modèle de la démocratie constructive ne relève pas d'une médiation ou d'une facilitation classique, du moins à nos yeux, et ne repose pas sur la recherche systématique de consensus, comme dans la visée 'harmonique' à la Habermas. Il n'implique pas non plus d'endosser tous les présupposés de la littérature agonistique, celle de Honneth (2000) ou de Mouffe (1999) par exemple, dans leur critique du modèle dialogique-consensuel, à rapprocher de Simmel (1995). En effet, le modèle de la démocratie constructive s'intéresse aux opportunités politiques que peuvent offrir en contexte telle ou telle option, l'entente ou le conflit, et aux possibilités de les articuler dans un processus de gouvernance. Il n'y donc pas dans ce modèle une vision de la politique qui opterait par principe et en général pour le conflit plutôt que pour l'entente ; mais ce qui est sûr, c'est que la vision politique sous-jacente n'opte pas uniquement pour l'entente. Pour un renvoi dos à dos des modèles harmoniques et agonistiques, voir Lavelle (2007).

${ }^{22}$ Il s'agit pour Rosanvallon (2006) de mettre en place des «modes de structuration intermédia ire (...) entre le pouvoir purement informel de l'opinion ou de l'intervention militante et le dispositif strictement constitutionnel». Il mentionne les actions de surveillance menées par «des organisations militantes non partisanes » telles que des agences citoyennes de notation ou des observatoires citoyens. Car c'est sur le terrain de l'expertise et de la veille citoyennes que se situe selon lui «l'un des enjeux essentiels du progrès démocratique »p. 306. voir également Rosanvallon (2008). 
La démocratie constructive, en tant que modèle politique normatif, s'emploie à favoriser l'émergence d'une forme de vie structurante pour les acteurs sociaux susceptible de garantir leur autonomie. Dans une forme de vie structurante, il est possible pour eux de faire une expérience quasi-politique ou sub-politique (au sens de la politique par le bas) de l'égalité, de la liberté, de l'engagement ou de l'implication. Il s'agit d'une expérience, dans une forme de vie quotidienne, d'une activité qui permet une articulation et une intégration de rôles sociaux généralement considérés comme distincts dans leur principe. Il s'agit des rôles sociaux de travailleur, de citoyen, de consommateur, de parent, etc., qu'un certain type d'organisation du travail et de la société maintient dans un rapport de désarticulation et de désintégration. Il semble ainsi que certaines formes de vie structurantes rendent possibles plus que n'importe quel dispositif institutionnel de délibération ou de participation l'articulation et l'intégration de ces différents rôles sociaux. C'est le cas par exemple dans des organisations de travail de type coopératif où l'articulation avec des visées citoyennes est assez nette, ne serait-ce qu'en vertu du principe d'égalité des travailleurs ${ }^{23}$.

C'est ainsi notamment qu'il est permis de sortir de la dépendance et de la polarisation entraînée par la démocratie d'interaction, qui risque de confiner les acteurs de la société dans une forme de démocratie de réaction à l'égard du pouvoir. A l'opposé, dans une démocratie de proaction, la société se prend en main et se donne la possibilité d'avoir une expérience politique qui lui est propre $^{24}$. Ce genre de conception de la politique, qui s'appuie sur la dynamique constructive, créative et expérimentale de la société, échappe semble-t-il au reproche de démocratie impolitique, que Rosanvallon distingue de l'apolitique ${ }^{25}$. Ainsi, la conception de la démocratie constructive, en promouvant une expérience politique enracinée dans une forme de vie, une communauté ou un territoire, crée les conditions pour la construction d'un monde commun concret.

\section{Un dispositif de gouvernance de la technique relevant de la démocratie constructive}

Le projet CIP ('Cowam-in-Practice') concerne la gouvernance des activités de gestion des déchets radioactifs en Europe ${ }^{26}$. Il s'inscrit dans le cadre d'une réflexion participative engagée depuis une dizaine d'années dans le cadre du programme cadre de recherche Euratom de la Commission Européenne. Il s'appuie sur un dispositif méthodologique qui

\footnotetext{
${ }^{23}$ On peut se référer en France au statut légal d'entreprise coopérative connue sous le nom de SCIC (Société Coopérative d'Intérêt Collectif) qui offre la possibilité de faire une expérience, dans la sphère «privée » du travail, d'une égalité, d'une liberté et d'une autonomie entre personnes, qui sont en même temps producteurs et citoyens. Sur ce point, voir le site des SCOP (Société Coopératives et Participatives) : http://www.scic.coop. Leur slogan est assez explicite : "La démocratie nous réussit... ».

${ }^{24}$ La démocratie d'interaction laisse dans l'ombre, en effet, la question de la structure politique de la relation entre acteurs (le pouvoir et la société). Or, l'asymétrie de la structure de relation est une source potentielle de domination, mais aussi de frustration politique pour les acteurs de la société civile. Leur énergie politique investie dans la délibération et la participation peut en effet se trouver détournée ou déçue, au regard de l'impossibilité par ce biais de changer la logique d'un système.

${ }^{25}$ Idem.

${ }^{26}$ Pour une présentation de l'expérience d'inclusion de la société civile et de recherche coopérative dans les activités à risque et la gestion des déchets nucléaires, voir Heriard Dubreuil et Baudé (2008), Hériard Dubreuil et Gadbois (2005).
} 
tente de répondre aux multiples difficultés rencontrées depuis plusieurs décennies par les processus de décisions techniques et sociaux complexes de la gestion des déchets radioactifs (GDR) dans l'ensemble des pays concernés ${ }^{27}$. Le projet CIP suggère la voie d'une gouvernance réflexive et coopérative durable qui s'inscrit dans un modèle politique émergent de démocratie constructive.

\section{La description du dispositif CIP}

Le projet CIP propose un dispositif transitoire d'une durée déterminée (3 ans) qui a vocation à susciter une transformation durable de la gouvernance de la GDR sur le long terme ${ }^{28}$. Il crée les conditions d'un renforcement de l'autonomie des acteurs concernés de la société à l'égard des institutions, en privilégiant un idéal démocratique d'auto-organisation et d'auto-apprentissage. Le projet européen CIP s'est proposé d'atteindre ces objectifs en créant pour trois ans dans chacun des cinq pays participants (Espagne, France, Roumanie, Royaume-Uni, Slovénie) un processus impliquant : un groupe de porteurs d'enjeux nationaux et locaux, avec le soutien méthodologique d'un facilitateur national, et le support d'une équipe méthodologique d'experts rassemblant des chercheurs et des spécialistes de différentes disciplines. La mise en oevre du dispositif comprend deux étapes fondamentales :

- Etape 1: Elle consiste à identifier les représentants des différentes catégories dacteurs concernés par la problématique de la GDR (opérateurs industriels, autorités publiques, experts, élus territoriaux, associations, individus concernés,...). Le rôle du facilitateur national et du président du groupe national est alors de proposer à ces acteurs une démarche de recherche coopérative et de négocier avec ceux-ci les conditions de leur participation. C'est aussi de préciser les règles d'interaction dans le cadre du processus qui comprend des institutions temporaires et des ressources spécifiques (cognitives, financières, etc.). Les acteurs non institutionnels sont ici dans une posture politique qui dépasse le cadre du processus temporaire, ce en quoi il ne s'agit pas d'un panel d'acteurs de la société. Les ressources du dispositif sont destinées notamment à créer les conditions d'un rééquilibrage des positions nécessairement inégales des différents acteurs. Par exemple, en mettant des ressources d'expertise européenne indépendante à la disposition des acteurs de la société.

- Etape 2: Elle consiste à créer les conditions pour que les différents acteurs identifient et mettent à l'agenda du groupe des questions importantes à leurs yeux dans le contexte de la GDR, en dépit des divergences. Il faut bien noter que le processus ne vise pas en priorité à faire émerger des points d'entente ou de consensus, mais plutôt à initier un chemin d'investigation des questions identifiées dans des conditions acceptables et équitables pour les différentes parties. A partir de là, le processus peut prendre des formes différentes selon les cas. La recherche coopérative peut consister à rassembler sur une question une pluralité de perspectives et à créer les conditions d'un dialogue critique dans le groupe plénier qui constitue, de par la pluralité de ses membres et la présence de représentants institutionnels, une forme d'espace public. Il peut également comprendre des investigations spécifiques de la part des acteurs de la société civile,

\footnotetext{
${ }^{27}$ Gilles Hériard Dubreuil (2000).

${ }^{28}$ Pour plus d'informations sur le projet CIP, on peut se reporter au rapport CIP (2010).
} 
en amont du dialogue du groupe national (plénier), avec le support des ressources cognitives et financières pour rééquilibrer leur position. C'est ainsi, par exemple, que des investigations spécifiques des acteurs territoriaux ont été menées dans le contexte français, sur la question de la réversibilité.

\section{L'examen du processus CIP}

Un processus coopératif d'examen de l'impact du projet CIP a été réalisé de façon intégrée dans le cadre du projet. L'objectif était d'en tirer le retour d'expérience des cinq processus nationaux et de formuler des recommandations susceptibles d'être mises en oevre dans l'ensemble des pays de l'UE. Ce processus d'examen a été mené de la façon suivante : un membre de l'équipe de recherche, spécifiquement dédié à cette fonction d'évaluation, a d'abord pu participer en tant qu'observateur aux principales réunions du projet depuis sa phase initiale. Vers la fin de la seconde année du projet, celui-ci a réalisé une enquête d'évaluation approfondie dans chacun des pays concernés auprès d'une pluralité de participants des groupes de porteurs d'enjeux nationaux et locaux. Les résultats de cette enquête ont ensuite fait l'objet d'un travail d'analyse réflexive et d'interprétation pluridisciplinaire par l'équipe de recherche européenne du projet. Les résultats de ces travaux ont été ensuite présentés et discutés dans un cadre pluraliste dans les instances de gouvernance de ce projet de recherche. Il en ressort un certain nombre de points fondamentaux.

Tout d'abord, les participants interrogés reconnaissent que CIP a constitué un espace de discussion démocratique différent d'autres lieux jugés plus formels. Ce cadre a permis une sorte d'acculturation démocratique, une familiarisation et une assimilation de règles du jeu différentes, l'exploration d'un autre système de relations possibles entre acteurs. Il apparaît dans les entretiens que le processus CIP a permis à certains acteurs qui ne le faisaient pas auparavant de faire entendre leur voix, favorisant une parole et une écoute pour des acteurs marginalisés dans ces processus techniques de gestion et de décision. CIP a favorisé une prise de conscience des enjeux de la situation par certains acteurs qui ont pu ainsi découvrir des aspects techniques difficiles et en général peu abordés. Certains acteurs appartenant aux localités concernées par la GDR ont pris conscience des leviers d'action dont ils disposaient ou dont ils manquaient. Cependant, ces mêmes acteurs soulignent que le projet CIP ne leur a pas permis d'avoir un une influence directe sur les décideurs.

Les acteurs de CIP de tous bords témoignent d'une grande lucidité dans leur analyse des rapports stratégiques dans le champ de la GDR. Le processus a permis de rendre visible les jeux d'acteurs et des enjeux non explicités auparavant et a encouragé chez certains acteurs locaux des postures nouvelles qui leur ont permis d'expérimenter un jeu stratégique différent. Il a contribué dans plusieurs contextes à un rééquilibrage de l'influence des collectivités territoriales, particulièrement celles qui se trouvaient dans une position de soumission, voire d'écrasement, parfois liée à une histoire politique bien antérieure. Les raisons de l'émergence de nouvelles catégories d'acteurs en posture active dans le contexte de CIP résultent notamment du support heuristique, parfois stratégique, apporté par le projet. Sans aucun doute, la plupart des acteurs estiment avoir une vision plus claire du processus de gouvernance de la GDR, même s'il demeure de nombreuses incertitudes sur son avenir. CIP est identifié comme un lieu d'apprentissage pour les acteurs, en particulier les plus inexpérimentés, dans la compréhension et l'analyse de processus techniques et sociaux complexes. 
Par exemple, en Roumanie, le maire d'une commune proche d'un site destiné à héberger des déchets radioactifs considère que le processus CIP a «changé sa vie », selon ses propres termes. Il a pu en effet prendre conscience des différents leviers d'action qui s'offraient à lui, notamment au plan du droit communautaire européen, et sortir ainsi d'une situation critique de soumission aux autorités publiques. En revanche, le maire de l'autre commune voisine, de taille beaucoup plus importante, et qui abrite l'une des centrales nucléaires du pays, porte un jugement moins enthousiaste sur ce processus. Issu du mouvement associatif oppositionnel, mais maintenant maire d'une ville nucléaire, il regrette de n'avoir pas pu accéder directement aux décideurs-clés du pays grâce au dispositif CIP, ce qui constituait son attente majeure. Il faut dire que CIP a également permis de mettre à jour des risques d'asymétries de pouvoirs et de ressources entre les deux localités, tant au plan financier que décisionnel. Il a produit ainsi une forme de rééquilibrage heuristique et stratégique entre ces deux acteurs, ainsi qu'entre un de ses acteurs et les autorités nationales en charge des déchets radioactifs.

\section{Un exemple thématique : la transformation du cadrage problématique en matière $d$ réversibilité dans le cas de la gestion des déchets radioactifs en France}

La loi de 2006 sur la gestion des déchets radioactifs propose un concept de stockage réversible en couche géologique profonde ${ }^{29}$. La réversibilité doit être définie par le Parlement en 2016, mais reste encore relativement ouverte aujourd'hui. Ce thème a été identifié comme un sujet majeur par le groupe national des porteurs d'enjeux, mis en place en 2007 dans CIP. Les participants - acteurs locaux et représentants des principales institutions nationales concernées par cette question - ont constitué un ensemble pluraliste disposant de compétences et d'expériences diversifiées. Les institutions ont présenté leurs réflexions sur la réversibilité sur un plan technique, réglementaire et financier. L'ANCLI (Association Nationale des Commissions Locales d'Information) a engagé autour des installations nucléaires en amont des travaux du groupe une réflexion des acteurs locaux basée sur leur propre retour d'expérience, avec un soutien méthodologique de CIP. Le principe d'une vigilance territoriale apparaît essentiel pour les acteurs locaux car l'impact du site se situe dans une perspective de très long terme.

Il apparaît que les contributions aboutissent à un transformation du cadrage du problème de la réversibilité. La réversibilité pratique, à la différence de la réversibilité théorique, se présente comme le maintien d'une capacité de choix entre retrait des colis, la poursuite d'un stockage réversible, ou la fermeture du stockage. Elle engage ainsi un ensemble de dimensions politiques, économiques, financières, éthiques et techniques et suppose l'existence effective des moyens appropriés sur ces différents plans dans un contexte démocratique et leur maintien durant la période de réversibilité. La définition de la réversibilité par le Parlement devra prendre en compte toutes ces composantes, plusieurs participants soulignant que la définition technique devrait découler du sens que l'on souhaite donner à ce concept, et non l'inverse. Un ensemble d'acteurs locaux et nationaux seront amenés à coopérer dans l'avenir pour définir cette capacité de choix et ses modalités.

\footnotetext{
${ }^{29}$ Pour une information plus complète sur l'histoire politique et technique de la gestion des déchets radioactifs, on peut se référer à Yannick Barthe (2006) ainsi qu'à Jean-Claude Petit (2000), ainsi que Le Dars (2004).
} 
Au final, on peut suggérer une interprétation en trois temps du processus CIP :

(1) Les acteurs étaient globalement d'accord pour construire ensemble les questions communes, ce en dépit des divergences manifestées qui portaient aussi bien sur la méthode suivie, le contenu des thèmes abordés, que les solutions proposées.

(2) Le rôle joué en amont par les médiateurs nationaux et les relations nouées avec CIP ont permis auprès des acteurs le déclenchement et la réalisation du processus proprement dit.

(3) CIP est conçu comme un processus temporaire, destiné à faciliter la gouvernance dans le long terme, et se voit doter dans l'ensemble d'un potentiel de transformation de la situation.

\section{Conclusion}

L'introduction de dispositifs dialogiques de délibération et de participation pour compléter les dispositifs plus classiques de représentation ou de délégation sont censés constituer une innovation en matière de gouvernance. Cependant, ils demeurent polarisés par la référence aux modèles dialogiques qui privilégient, à des degrés divers selon les auteurs ou les courants, la voie de la discussion et de l'entente.

Il existe sans nul doute un tropisme procédural de ces modèles et dispositifs dialogiques qui tendent à négliger la fonction déterminante des paradigmes, cadres de références, habitus ou arrière-plans (Background) des acteurs. Cette négligence constitue assurément un point aveugle de la théorie et de la pratique de la gouvernance démocratique en général et de la gouvernance de la technique en particulier. C'est précisément ce genre de point aveugle que la conception de la démocratie constructive, élaborée dans un rapport direct et étroit avec plusieurs terrains d'expérimentation dans un domaine technique précis, propose de réfléchir et si possible de combler. De ce point de vue, le domaine technique de l'industrie nucléaire, et en particulier celui de la gestion des déchets radioactifs, apparaît plus encore que d'autres domaines comme un terrain décisif entraînant une mise à l'épreuve des dispositifs se réclamant des modèles dialogiques. Il est aussi une mise à l'épreuve pour le modèle de la démocratie constructive présenté comme une alternative, ou du moins, un complément au dialogisme.

En effet, s'il advenait que dans un tel domaine, caractérisé par une culture de l'expertise et une tendance à la domination fortes, les modèles et dispositifs dialogiques permettaient une transformation significative et satisfaisante du système de relations entre acteurs, il ne serait d'aucune utilité de proposer une voie alternative. Or, on cons tate dans le champ technique de la gestion des déchets radioactifs une prédominance du modèle de la «technocratie éclairée », qui s'accommode fort bien d'une interaction dialogique ponctuelle avec des acteurs de la société civile, dans le genre du débat public ou de la conférence de citoyen. Ainsi, dans la GDR le couple Technocratie / Dialogique occupe le devant de la scène tout en se révélant dans l'incapacité de provoquer des changement effectif de gouvernance.

Il en va tout autrement lorsque les acteurs de la société civile sont soutenus par une structure et un processus de gouvernance coopérative et réflexive durable que l'on peut rattacher au type de la démocratie constructive. L'introduction d'un dispositif s'apparentant à la démocratie constructive, prenant en compte les conditions d'arrière plan, fait émerger les questionnements, des divergences et des conflits latents et démontre par là même leur existence et la nécessité de leur prise en compte par une véritable transformation de la gouvernance. Il est alors question 
d'une organisation de la montée en puissance et en compétence progressive des acteurs de la société civile, lesquels peuvent développer à l'occasion leur propre expertise. Leurs initiatives et leurs interventions démocratiques, articulée à une visée d'autonomie, peuvent dès lors aller bien au-delà des événements dialogiques dépendant d'une organisation institutionnelle de la délibération et de la participation.

La démocratie constructive n'est certainement pas une panacée pour la gouvernance de la technique, et elle n'est d'ailleurs jamais assurée de son succès, ne serait-ce qu'en raison de son caractère quelque peu déstabilisant pour les équilibres en place. Il arrive que, dans tel ou tel contexte local ou national, elle ne parvient pas à transformer de façon significative et satisfaisante le système de relations entre acteurs, certains ne voyant pas suffisamment d'attrait à un tel changement. Cependant, il est manifeste qu'elle entraîne, par un effet de renforcement des acteurs partenaires de la relation de coopération, une nouvelle posture politique de leur part ainsi qu'une consolidation de leur engagement.

Du côté des partenaires institutionnels, experts ou décideurs, une telle expérience leur révèle, sans que cela entraîne nécessairement pour eux un risque majeur, un champ de possibilités en matière de coopération avec la société civile qu'ils ne soupçonnaient pas auparavant. Du côté des partenaires citoyens ou associatifs, une telle expérience leur révèle un champ de possibilités en matière de coopération avec les experts et décideurs, mais aussi en matière d'initiative sociale autonome, qu'eux non plus ne soupçonnaient pas auparavant. Le révélateur du pouvoir social des citoyens, associations et autres organisations non gouverne mentales est sans nul doute leur capacité à créer un précédent dans leur relation avec les organes d'expertise. Leur influence peut se traduire par une transformation du cadre problématique, des cadres heuristique et stratégique, mais aussi, à un niveau plus profond relevant du paradigme des acteurs, du «cadre herméneutique » dans lequel ils puisent les modalités d'interprétation de leurs actions.

Quoi qu'il en soit, il apparaît dans la démocratie constructive que, au delà des conditions d'émergence, les conditions de résilience des processus de gouvernance coopérative et réflexive durable tiennent, non pas à des institutions, mais à des hommes et des femmes qui ont fait une certaine expérience de transformation de leurs cadres cognitifs et normatifs. La démocratie constructive n'a pas d'autre ambition que de contribuer, dans les limites qui sont les siennes, à une telle transformation, dans le champ de la gouvernance de la technique, ou dans d'autres champs de la gouvernance.

Sylvain Lavelle est au Centre Ethique, Technique et Société (CETS); Gilles Hériard Dubreuil et Serge Gadbois sont au Mutadis; Claire Mays est au Symlog; et Thierry Schneider est au Centre d'étude sur l'Evaluation de la Protection dans le domaine Nucléaire (CEPN). Cet article est issu de la recherche menée par les auteurs au sein d'un projet européen Cowam-in-Practice (CIP) financé par Euratom dans le cadre du programme FP6.

\section{Bibliographie}

Barber B. (1997) Démocratie forte, Paris, Desclée de Brouwer.

Barthe Y. (2006) Le pouvoir d'indécision, Paris, Economica.

Blondiaux L. (2008) Le nouvel esprit de la démocratie, Paris, Seuil.

Callon M., Lascoumes P., Barthe Y. (2001) Agir dans un monde incertain, Paris, Seuil. 


\section{Lavelle, Dubreuil, Gadbois, Mays et Schneider}

Callon M. (1998) « Des différentes formes de démocratie technique », Annales des mines, $\mathrm{n}^{\circ}$ 9, p. 63-73.

Dewey J. (2003) Le public et ses problèmes, Pau, Farago.

Feenberg A. (2004) Repenser la technique, Paris, La Découverte.

Habermas J. (2006) Idéalisations et communication, Paris, Fayard.

Habermas J. (1997) Droit et démocratie, Paris, Gallimard.

Habermas J. (1991) De l'éthique de la discussion, Paris, Flammarion.

Habermas J. (1987) Théorie de l'agir communicationnel, Paris, Fayard.

Heriard Dubreuil G. (2000) « Comment mener une politique de très long terme : le cas des déchets nucléaires »

Esprit, 269, p. 81-97.

Heriard Dubreuil G., Baude S. (2008) «Innovative Approaches to Stakeholder Involvement in Risk Governance.

Lessons from TRUSTNET IN ACTION European Research Project», - in European Risk Governance, CONNEX

Report Series Nr 06 Manheim http://www.connex-network.org/series

Gadbois S., Heriard Dubreuil G. (2005) «Local Stakeholder involvement in the perspective of nuclear waste management: Lessons from the COWAM network», Radioprotection, Suppl. 1, vol. 40, p. S959 à S965.

Held D. (2007) Models of Democracy, Cambridge, Polity Press.

Hermet G. (2005) La gouvernance, Paris, Karthala.

Honneth A. (2000) La lutte pour la reconnaissance, Paris, Cerf.

Latour B. (1999) Politiques de la nature, Paris, La Découverte.

Lavelle S. (2007) «La politique de la discussion», M. Revel, C. Blatrix, L. Blondiaux, J.-M. Fourniau, B. Hériard

Dubreuil, R. Lefebvre (dir.), Le Débat public : une expérience française de démocratie participative, Paris, La découverte, p. 353-366.

Lavelle S. (2006) Science, technologie et éthique, Paris, Ellipses.

Le Dars A. (2004) Pour une gestion durable des déchets nucléaires, Paris, PUF.

Lenoble J., Maesschalck M. (2010) Democracy, Law and Governance, Kluwer, Amsterdam.

Lenoble J., Maesschalck M. (2009) L'action des normes. Editions de l'Université de Sherbrooke.

Maesschalck M. (2001), Normes et contextes, Olms.

Moreau Defarges P. (2006) La gouvernance, Paris, PUF.

Mouffe C. (1999) « Deliberative democracy or agonistic pluralism», Social Research, Vol.66, no. 3, p. 745-758.

Petit JC. (2000) Le stockage des déchets radioactifs, Thèse de Doctorat, Ecole des Mines de Paris

M. Revel, C. Blatrix, L. Blondiaux, J.-M. Fourniau, B. Hériard Dubreuil, R. Lefebvre (dir.), Le Débat public : une expérience française de démocratie participative, Paris, La découverte

Rosanvallon P. (2008) La légitimité démocratique, Paris, Seuil.

Rosanvallon P. (2006) La contre-démocratie, Paris, Seuil.

Sclove R. (2003) Choix technologiques, choix de société, Paris, Descartes et Cie.

Simmel G. (1995) Le conflit, Belval, Circé.

Winner L. (2002) La baleine et le réacteur, Paris, Descartes et Cie. 